OPEN ACCESS

Edited by:

Chaofeng Han,

Second Military Medical University,

China

Reviewed by:

Degang Yang,

Tongji University, China

Minghong Jiang,

China Academy of Chinese Medical

Sciences, China

*Correspondence:

Lu Fang

fanglu@medmail.com.cn

Fan Zhou

nczhoufan@hotmail.com

Bo Liang

Ib2087@163.com

${ }^{\dagger}$ These authors have contributed equally to this work

Specialty section:

This article was submitted to

Molecular Innate Immunity,

a section of the journal

Frontiers in Immunology

Received: 29 December 2021

Accepted: 10 January 2022

Published: 27 January 2022

Citation:

Zheng $H$, Zheng $W-j$,

Wang Z-g, Tao Y-p,

Huang $Z-p$, Yang $L$, Ouyang $L$,

Duan Z-q, Zhang $Y-n$, Chen $B-n$,

Xiang $D-m$, Jin G, Fang L, Zhou F and Liang B (2022) Decreased Expression of Programmed Death Ligand-L1 by

Seven in Absentia Homolog 2 in

Cholangiocarcinoma Enhances

T-Cell-Mediated Antitumor Activity.

Front. Immunol. 13:845193.

doi: 10.3389/fimmu.2022.845193

\section{Decreased Expression of Programmed Death Ligand-L1 by Seven in Absentia Homolog 2 in Cholangiocarcinoma Enhances T-Cell-Mediated Antitumor Activity}

\author{
Hao Zheng ${ }^{1,2,3,4,5 t}$, Wen-juan Zheng ${ }^{1 \dagger}$, Zhen-guang Wang ${ }^{3,4,5 t}$, Yuan-ping Tao ${ }^{6 t}$, \\ Zhi-ping Huang ${ }^{7 \dagger}$, Le Yang ${ }^{6}$, Liu Ouyang ${ }^{8}$, Zhi-qing Duan ${ }^{1}$, Yi-nuo Zhang ${ }^{1}$, \\ Bo-ning Chen ${ }^{1}$, Dai-min Xiang ${ }^{9}$, Gang Jin ${ }^{8}$, Lu Fang ${ }^{1 *}$, Fan Zhou ${ }^{1 *}$ and Bo Liang ${ }^{1 *}$ \\ ${ }^{1}$ Department of General Surgery, The Second Affiliated Hospital of Nanchang University, Nanchang, China, ${ }^{2}$ Department of \\ Reproductive Heredity Center, Changhai Hospital, Second Military Medical University, Shanghai, China, ${ }^{3}$ Third Department \\ of Hepatic Surgery, Eastern Hepatobiliary Surgery Hospital, Second Military Medical University, Shanghai, China, ${ }^{4}$ Key \\ Laboratory of Signaling Regulation and Targeting Therapy of Liver Cancer (SMMU), Ministry of Education, Shanghai, China, \\ 5 Shanghai Key Laboratory of Hepatobiliary Tumor Biology (EHBH), Shanghai, China, ${ }^{6}$ National Liver Tissue Bank, Eastern \\ Hepatobiliary Surgery Hospital, Second Military Medical University, Shanghai, China, ${ }^{7}$ Department of Hepatobiliary Surgery, \\ General Hospital of Southern Theatre Command, Guangzhou, China, ${ }^{8}$ Department of Hepatobiliary Pancreatic Surgery, \\ Changhai Hospital of Second Military Medical University, Shanghai, China, ${ }^{9}$ State Key Laboratory of Oncogenes and Related \\ Genes, Shanghai Cancer Institute, Renji Hospital, Shanghai Jiao Tong University School of Medicine, Shanghai, China
}

N6-methyladenosine (m6A) has been reported as an important mechanism of posttranscriptional regulation. Programmed death ligand 1 (PD-L1) is a primary immune inhibitory molecule expressed on tumor cells that promotes immune evasion. In addition, seven in absentia homolog 2 (Siah2), a RING E3 ubiquitin ligase, has been involved in tumorigenesis and cancer progression. However, the role of m6A-METTL14Siah2-PD-L1 axis in immunotherapy remains to be elucidated. In this study, we showed that METTL14, a component of the $\mathrm{m}^{6} \mathrm{~A}$ methyltransferase complex, induced Siah2 expression in cholangiocarcinoma (CCA). METTL14 was shown to enrich $\mathrm{m}^{6} \mathrm{~A}$ modifications in the 3'UTR region of the Siah2 mRNA, thereby promoting its degradation in an YTHDF2-dependent manner. Furthermore, co-immunoprecipitation experiments demonstrated that Siah2 interacted with PD-L1 by promoting its K63-linked ubiquitination. We also observed that in vitro and in vivo Siah2 knockdown inhibited T cells expansion and cytotoxicity by sustaining tumor cell PD-L1 expression. The METTL14Siah2-PD-L1-regulating axis was further confirmed in human CCA specimens. Analysis of specimens from patients receiving anti-PD1 immunotherapy suggested that tumors with low Siah2 levels were more sensitive to anti-PD1 immunotherapy. Taken together, our results evidenced a new regulatory mechanism of Siah2 by METTL14-induced mRNA epigenetic modification and the potential role of Siah2 in cancer immunotherapy.

Keywords: N6-methyladenosine, METTL14, Siah2, PD-L1, immunotherapy 


\section{INTRODUCTION}

Cholangiocarcinoma (CCA) is the second most common primary malignant tumor of liver cancers, whose recurrence rate is significantly higher than that of hepatocellular carcinoma. The overall median survival time of ICC patients is less than one year (1-3). For patients with advanced or inoperable CCA, gemcitabine-cisplatin combination is recommended as systemic therapy but with marginal improvement in overall survival. Unfortunately, we currently lack of a no standard second-line therapy (4). Thus, it is urgent to develop new strategies to prevent tumor progression and improve the prognosis of CCA patients.

RNA N6-methyladenosine (m6A) is the most abundant internal modification in mRNAs, which is widely present in mammal cells and mainly present in the last exon of mRNA (5). Its methylation is regulated by "writer," "eraser," and "reader" proteins (6). In addition, m6A methyltransferase complex is mainly composed of METTL3 (methyltransferase-like 3), METTL14 (methyltransferase-like 14), and WTAP (Wilms tumor 1-associated protein), which act as "writer" to catalyze methylation processes, whereas FTO (fat mass and obesity associated protein) and ALKBH5 (alkB homolog 5) act as demethylases ("eraser"). The ultimate fate of m6A methylated mRNA depends on the "reader" (e.g., YTHDF1, YTHDF2, and YTHDF3) that recognizes them, affecting mRNA translation, stability, splicing, and nuclear transportation (7). Numerous m6A targets have been involved in cell tissue development and stem cell self-renewal and differentiation, circadian rhythm regulation, T-cell homeostasis, mouse fertility, postnatal development of the mouse cerebellum, innate immune response, ultraviolet-induced DNA damage response, and dendritic cell antigen presentation $(8-10)$. However, the possible role of $\mathrm{m} 6 \mathrm{~A}$ modification in tumor-immune microenvironment and in CCA remains to be elucidated.

Programmed death-ligand 1 (PD-L1, also named B7-H1) is a major inhibitory immune checkpoint molecule that facilitates tumor evasion of immune surveillance by binding to programmed death receptor-1 (PD-1) on $\mathrm{T}$ cells, which induces $\mathrm{T}$ lymphocytes apoptosis, anergy, and functional exhaustion (11). The use of anti-PD-1/PD-L1 immunotherapy to block such an interaction, activating T-cell immunity has produced significant results in various types of cancer (12). In this regard, the anti-PD-1 antibody pembrolizumab has been approved by the FDA for the treatment of patients with unresectable or metastatic mismatch repair deficient and/or microsatellite-high solid tumors, including CCA, which progressed after prior therapy (13). The objective response rate of pembrolizumab is still limited but a better outcome was associated with high PD-L1 expression in CCA (14). Understanding the regulation mechanism underlying PD-L1 expression is essential for developing efficient combination immunotherapeutic strategies (15). It has been reported that PD-L1 expression is regulated by genetic alterations (e.g., rearrangements in the $30 \mathrm{UTR}$ PD-L1 mRNA), tumor-intrinsic oncogenic pathways (e.g., RAS-MEK-ERK, CDK5, PI3K-AKT, HIF-1a), and posttranslational modifications (e.g., CMTM6/
CMTM4, HIP1R, CUL3), whereas the exact regulation mechanism of PD-L1 on protein level is still unknown (16).

SIAH1 and Siah2 are highly homologous RING domain E3 ligases that often target common proteins for degradation (17). Two SIAH homologs in humans (SIAH1 and Siah2) and three homologs in mice (Siah1a, Siah1b, and Siah2) have been reported (18). SIAH proteins contain an N-terminal RING domain and a C-terminal substrate-binding domain (SBD) (19), which are involved in ubiquitination of diverse substrates and other biological processes such as RAS signaling, DNA damage, hypoxia, p38/JNK/NF-кB pathways, and transcription (20-22). In particular, Siah2 plays a critical role in tumorigenesis and cancer progression (23). Numerous studies have determined that Siah2 exerts its biological function, depending on the tumor development stage (23). However, the exact regulation mechanism of Siah2-regulated PD-L1 on protein level in CCA is still unclear.

In the present study, we showed an epigenetic regulating mechanism of Siah2 on mRNA level by METTL14, which depended on m6A modification. We further unveiled a complex role of Siah2 in immune microenvironment and demonstrated that tumors with low Siah2 expression pattern were more sensitive to anti-PD-1 immunotherapy. Taken together, our results evidenced a new regulation mechanism of PD-L1 and unveiled a role of METTL14-Siah2 axis in immunotherapy, which might contribute to CCA immunotherapy.

\section{MATERIALS AND METHODS}

\section{Patients and Tissue Specimens}

Tissue specimens were obtained from the Eastern Hepatobiliary Surgery Hospital (Shanghai, China) and the Changhai Hospital (Shanghai, China). Written informed consent was obtained from patients. The procedure of human specimen collection was approved by the Ethics Committees of these Hospitals.

\section{Lentiviral Transduction for Stable Cell Line}

Lentiviruses used in this study were purchased from Genechem (Shanghai, China). Lentiviral infection was performed according to manufacturers' instructions. In brief, RBE and HUCCT1 cells were seeded in 6-well plates in $2 \mathrm{~mL}$ complete medium and transduced by lentivirus at a multiplicity of infection of 15 to 20 in the presence of the HitransG A reagent (Genechem). We then used $5 \mathrm{~g} / \mathrm{mL}$ puromycin (Selleck, Shanghai, China) for the selection of transduced cells. After continuous selection for approximately two weeks, the surviving cell colonies were expanded. The efficacy of overexpression and knockdown was verified by Western blotting.

\section{shRNA Transfection}

shRNAs were synthesized by Biotend Biotechnology (Shanghai, China). For siRNA transfection, RBE and HUCCT1 cells were cultured in 12 - or 6 - well plates to $60 \%-70 \%$ confluency. One hundred nanomoles of siRNA or siRNA negative control (NC) were transfected to cells using Lipofectamine 2000 (ThermoFisher Scientific, Waltham, MA, USA), following manufacturer's 
instructions. After $48 \mathrm{~h}$ or $72 \mathrm{~h}$ of transfection, cells were used for subsequent experiments. Sequences of shRNA seed sequence were provided in Table $\mathbf{S 1}$.

\section{Western Blot}

Western blotting analysis was performed as previously reported (24). In brief, cells were lysed in RIPA lysis buffer (Beyotime Biotechnology, Shanghai, China) with $1 \mathrm{mM}$ PMSF on ice for 10 min. Protein concentrations were measured by the Pierce ${ }^{\mathrm{TM}}$ BCA Protein Assay Kit (ThermoFisher Scientific). Equal amounts of protein were separated by SDS-PAGE and transferred onto 0.22 $\mu \mathrm{m}$ nitrocellulose membranes (Millipore, Cork, Ireland). Membranes were then incubated with anti-primary antibodies (LI-COR Biosciences, Lincoln, NE, USA) overnight at $4^{\circ} \mathrm{C}$, followed by incubation with IRDye 800 goat anti-rabbit antibodies (LI-COR Biosciences) for $1 \mathrm{~h}$ at room temperature. After removing the unbound antibodies, the labelled bands were scanned in the Odyssey ${ }^{\circledR}$ CLx Infrared Imaging System (LI-COR Biosciences). Anti-primary antibodies are listed in Table S2.

\section{RNA Isolation and Quantitative Real Time PCR (RT-PCR)}

TRIzol $^{\text {TM }}$ Reagent (Invitrogen, Carlsbad, CA, USA) was used to isolate total RNA from samples. Quantitative RT-PCR was performed as previously reported (25) on an ABI 7300 Fast RealTime PCR System (Applied Biosystems, Foster City, CA), using the SYBR Green PCR kit (Applied TaKaRa, Otsu, Shiga, Japan). The expression levels of the target genes were normalized against GAPDH and analyzed by the Delta-Delta-Ct $(\triangle \triangle \mathrm{Ct})$ method (3). Primers for quantitative RT-PCR are listed in Table S3.

\section{Nuclear and Cytoplasmic Fractions Assay}

Nuclear and cytoplasmic fractions were extracted by the PARISTM Kit (Ambion, Austin, TX, USA), following manufacturer's instructions and performed as previously reported (25). GAPDH and histone H3 (Bioss Antibodies Inc., Woburn, MA, USA) protein levels detection by Western blotting was used to confirm the successful cellular fraction.

\section{Cell Proliferation Assay}

Relevant cells were seeded in 96-well plates at a density of $1 \times 10^{3}$ cells/well. Cell viability was determined by the CCK- 8 assay kit, following manufacturer's instructions at indicated timepoints after seeding. Results were analyzed using the GraphPad Prism 8.0 software (GraphPad Software Inc., San Diego, CA, USA).

\section{Quantitative Analysis of Immunohistochemistry (IHC) Staining}

IHC was regularly performed and $\mathrm{H}$-Score was calculated for quantitative analysis of IHC staining, as previously reported (26). The photographs of the stained sections were obtained by the Leica Aperio AT Turbo Digital Slide Scanning System (Leica Biosystems, MA, USA).

\section{hPBMC+ Humanized mouse models}

hPBMC+ humanized NCG mice were purchased from the Model Animal Research Center of Nanjing University and were constructed as previously reported (25). Immune cell percentages were detected by flow cytometry 3 wk after hPBMC+ cell injection, hPBMC+ humanized NCG mice were randomly assigned into experiment groups. Indicated CCA cells with LV-NC/LV-SIAH2 of $5 \times 106$ were injected into the right flank of hPBMC+ humanized NCG mice. Tumor volume was calculated by the following formula: volume $=\mathrm{ab} 2 / 2$ ( $\mathrm{a}$, the longer axis; $b$, the shorter axis). After $35 \mathrm{~d}$ of cell inoculation, hPBMC+ humanized NCG mice were euthanized and tumorinfiltrating leukocytes were isolated and subjected to CyTOF analysis. The animal study was conducted in conformity with $\mathrm{NIH}$ and the Second Affiliated Hospital of Nanchang University, Servicebio Animal Welfare guidelines and approved by Wuhan Servicebio Technology Co., Ltd., China.

\section{PD-L1 Detection on Cell Surface}

PCCA\#1/\#2 Cells were digested and collected. After washing with staining buffer, $1 \times 10^{6}$ cells were suspended in $100 \mathrm{~mL}$ of staining buffer and incubated at $4^{\circ} \mathrm{C}$ for $30 \mathrm{~min}$ with PE Mouse Anti-Human PD-L1 (MIH1; BD Biosciences, San Jose, CA, USA) or APC Mouse Anti-Human PD-L1 (MIH1; BD Biosciences). After washing with staining buffer, samples were subjected to flow cytometry analysis with a BD LSRFortessa cell analyzer (BD Biosciences). Data were then analyzed with the FlowJo vX.07 software (Tree Star, San Carlos, CA, USA).

\section{RNA Immunoprecipitation}

RNA immunoprecipitation assay was conducted with the Magna RIP RNA-Binding Protein Immunoprecipitation Kit (Millipore), following manufacturer's instructions and performed as previously reported (25). RNA quality was evaluated on a Nanodrop2000 (Thermo Scientific, Wilmington, DE, USA).

\section{mRNA Stability Detection}

mRNA stability assays were performed as previously reported (25). Cells were cultured overnight, after which they were treated with $5 \mathrm{mg} / \mathrm{mL}$ actinomycin $\mathrm{D}$ at indicated times. Total RNA was extracted by TRIzol (Invitrogen). Quantitative RT-PCR was performed to determine the relative level of indicated mRNA.

\section{Isolation and Activation of Human Peripheral Blood Mononuclear Cells (HPBMC)}

HPBMC from health donors were provided by the Changhai Hospital (Shanghai, China) and isolated with Lymphoprep (Stemcell Technologies, Vancouver, Canada), following manufacturer's instructions and performed as previously reported (23).

\section{Statistical Analysis}

Statistical tests were two sided and values were expressed as mean $\pm \mathrm{SD}$, unless otherwise specified. The analysis was performed using the GraphPad Prism8 software (GraphPad Software Inc.). Unpaired Student $t$ test or paired Student $t$ test was used to compare between two experimental groups. Cumulative survival time was estimated by the Kaplan-Meier method, and the log-rank test was applied to compare groups. 
$\mathrm{P} \leq 0.05$ was considered statistically significant. We included all animal data.

Details of other regular experiments were provided in Supplementary Materials and Methods.

\section{RESULTS}

\section{Siah2 Is a Direct Target of METTL14- Induced m6A Modification in CCA}

We found that increased METTL14 caused a significant reduction on Siah2 protein levels in human CCA cell lines, whereas increased FTO, METTL3, or ALKBH5 did not affect its levels (Figure 1A). This indicated that Siah2 was specifically regulated by METTL14 but not by FTO, METTL3, or ALKBH5 in CCA. The fact that LV-METTL14 did not affect Siah2 protein degradation rate was demonstrated by the use of cycloheximide (CHX) and MG132 treatments (Figures 1B, C). Furthermore, we reversed LV-METTL14-restrained Siah2 protein and mRNA level, using the global methylation inhibitor 3-deazaadenosine (DAA) (Figures 1D, E), thus indicating that METTL14 controls Siah2 expression depending on its demethylation activity. The m6A level of Siah2 total mRNA was significantly increased in LV-METTL14 cells (Figure 1F).

The Siah2 m6A level was positively associated with METTL14 mRNA and negatively associate with Siah2 mRNA. METTL14 mRNA was negatively associated with Siah2 mRNA in $21 \mathrm{CCA}$ tissues (Figure 1G). Taken together, these data indicated that METTL14 regulated the level of m6A modification in CCA.
A
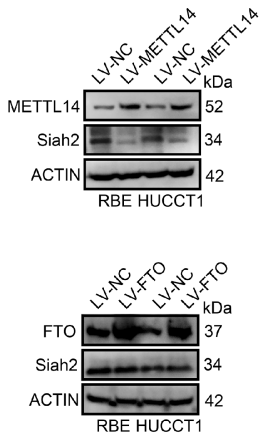

C

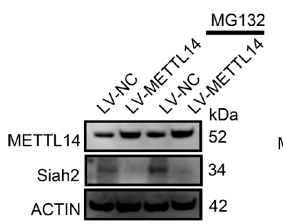

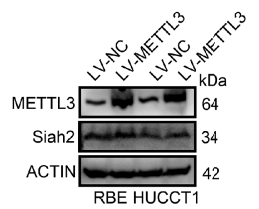

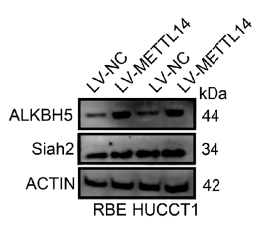

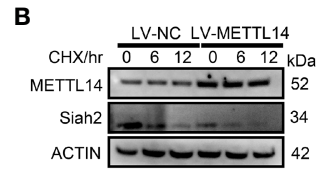
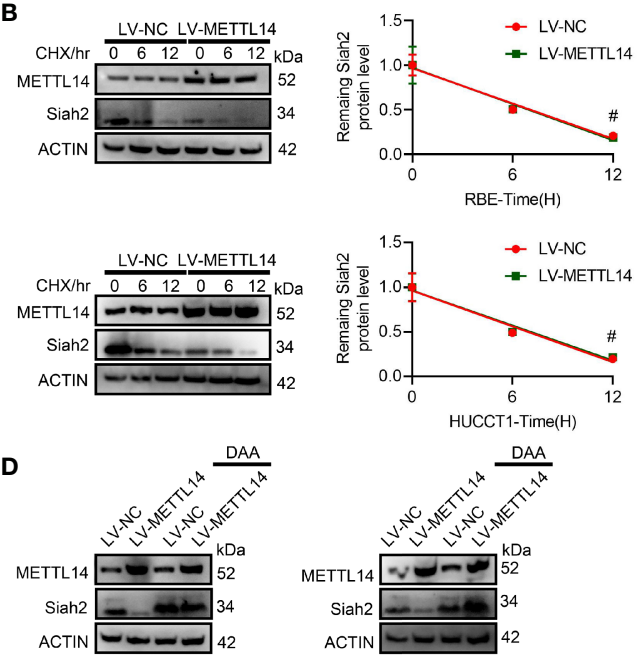

E
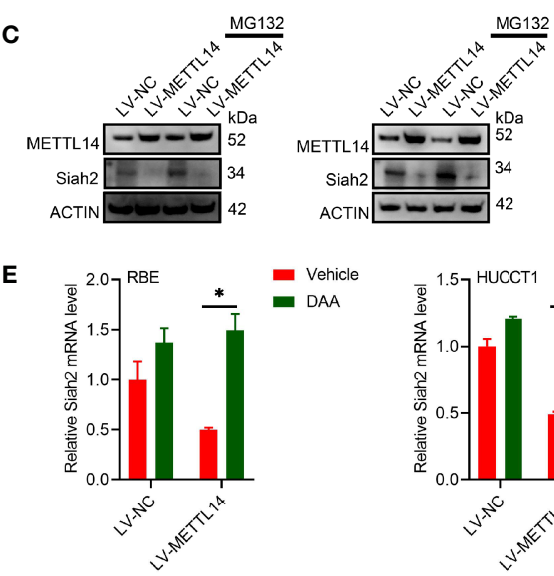

G
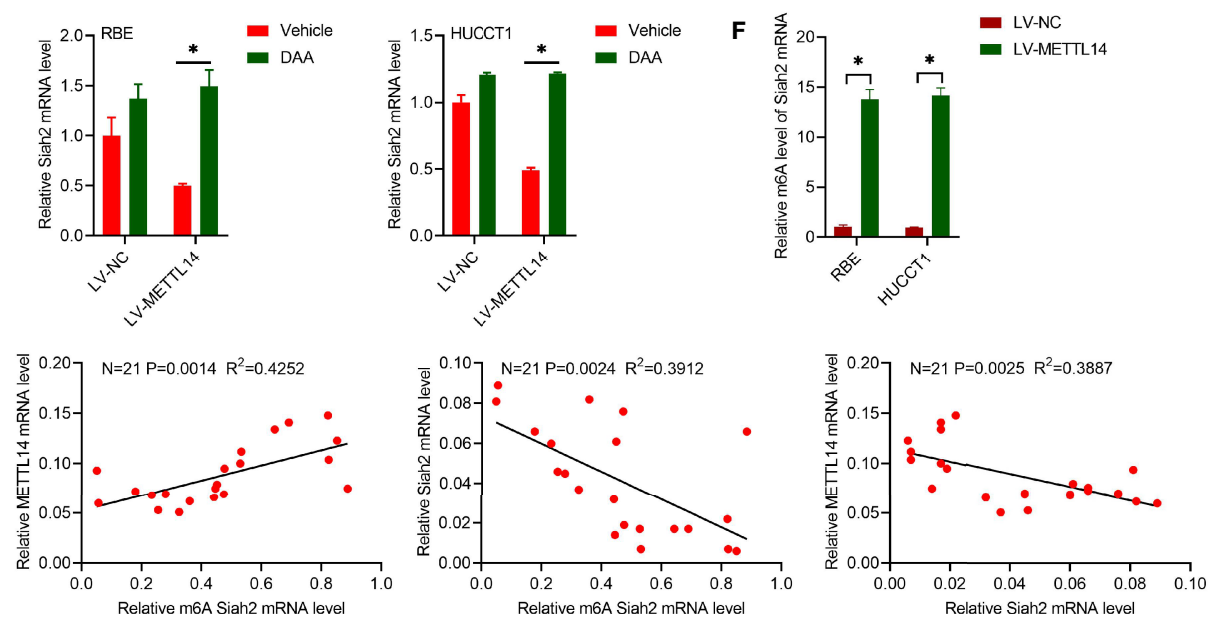

FIGURE 1 | Siah2 is a direct target of METTL14-induced m6A modification in CCA. (A), Western blot analysis for Siah2 expression in CCA cell lines with stable METTL14, METTL3, ALKBH5, or overexpressed FTO. (B), The rate of Siah2 protein degradation was checked by Western blotting in CCA cells with METTL14 knocked down upon CHX (protein synthesis inhibitor) treatment. (C). Western blot analysis for Siah2 expression in CCA cells with METTL14 knocked down upon MG132 treatment. (D, E), Western blot and RT-PCR analysis for Siah2 expression in CCA cells with LV-NC/-METTL14 upon DDA 0-25 mmol/L treatment for 24 h. (F), m6A fold enrichment of Siah2 total mRNA detected by MeRIP quantitative RT-PCR of fragmented mRNA with indicated primers. (G), Correlation between METTL14 MRNA level, M6A level of Siah2, and Siah2 MRNA level in CCA tissues. Mean \pm SEM of three independent experiments. ${ }^{*} P<0.05 ;{ }^{*} P>0.05$. 


\section{METTL14 Promotes Siah2 mRNA Degradation in CCA}

It has been reported that m6A modification affects mRNA stability of targeted mRNAs, depending on the distinct proteins (YTHDF1, YTHDF2, and YTHDF3) that recognize them. Blocking new RNA synthesis with actinomycin D indicated that METTL14-stable overexpression significantly increased Siah2 mRNA stability, whereas METTL14 knockdown inhibited Siah2 mRNA degradation (Figures 2A-C). Furthermore, METTL14 deficiency significantly increased and METTL14 overexpression significantly decreased the level of Siah2 mRNA in cytoplasmic and nuclear fractions (Figures 2D, E). YTHDF2 has been reported to specifically recognize and bind m6A-containing RNAs, regulating mRNA stability (25). As shown in Figures 2F-H, YTHDF2 knockdown significantly increased
Siah2 mRNA level and stability of PD-L1 mRNA. Following, the interaction between YTHDF2 and Siah2 mRNA was confirmed by the RIP assay (Figure 2I). Based on SRAMP (A sequence-based N6-methyladenosine (m6A) modification site predictor) database, seven peaks were identified in Siah2 mRNA, with peaks 6 to 7 on

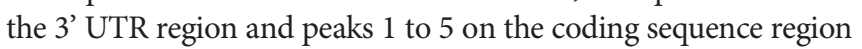
(Figure 2J). Notably, the RNA pull-down assays verified that YTHDF2 predominantly bound to the Siah2 $3^{\prime}$-UTR region, instead of CDS in CCA cells, and the specific binding was significantly impaired after m6A peak depletion(Figure 2K). These data suggested that METTL14 regulated the stability of Siah2 mRNA in an YTHDF2-dependent manner.

\section{Siah2 Regulates PD-L1 Stability in CCA}

It was found that protein level but not the mRNA level of PD-L1 increased in CCA cells with Siah2 knocked down (Figures 3A, B).
A

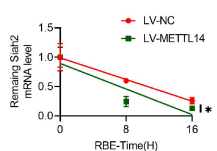

B

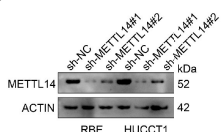

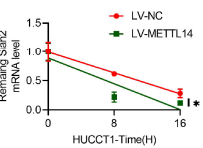

F

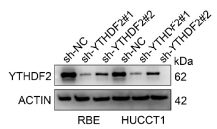

C
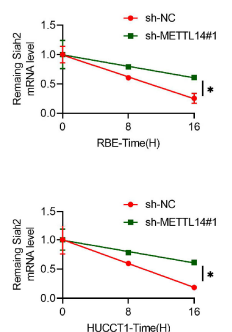

D
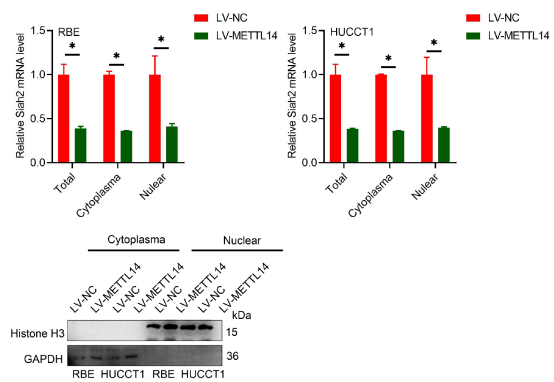

E

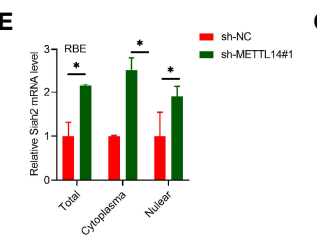

G

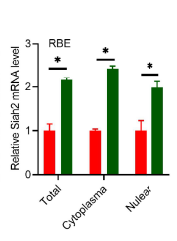

H

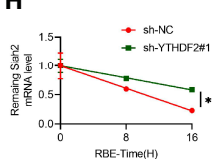

J

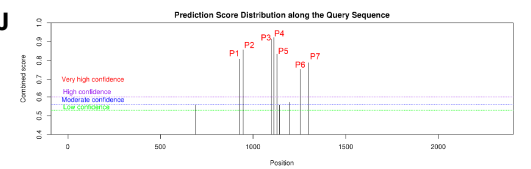

K

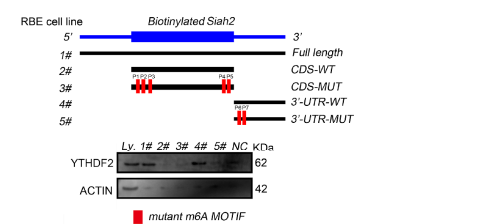

I
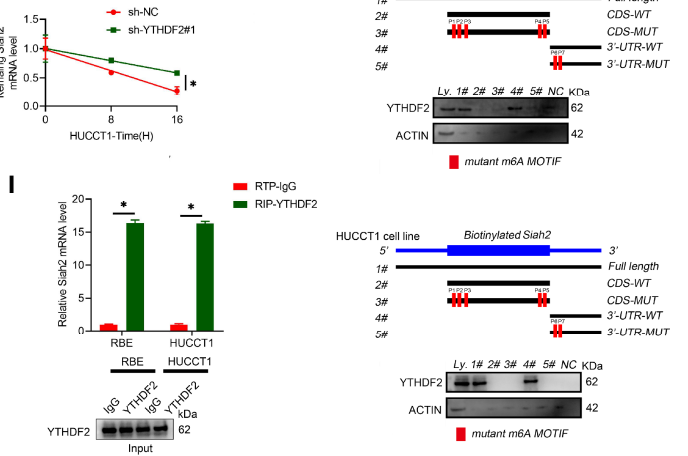

FIGURE 2 | METTL14 promotes Siah2 mRNA degradation in CCA. (A), The stability of Siah2 mRNA in CCA cells with LV-NV or LV-METTL14 was detected by quantitative RT-PCR upon actinomycin D $10 \mathrm{mg} / \mathrm{ml}$ treatment. (B), Western blot analysis for METTL14 expression in CCA cell lines with stable METTL14 knockdown. (C), The stability of Siah2 mRNA in CCA cells with sh-NC or sh-METTL14 was detected by quantitative RT-PCR upon $10 \mathrm{mg} / \mathrm{mL}$ actinomycin D treatment. (D, E), Quantification of Siah2 mRNA level in total, nuclear, and cytoplasmic fractions by quantitative RT-PCR in CCA cells. Nuclear and cytoplasmic fractions were evaluated by Western blot analysis. (F), The successful knockdown of YTHDF2 in CCA cells was confirmed by Western blot analysis. (G), The expression of Siah2 mRNA was detected by quantitative RT-PCR in CCA cells transfected with sh-NC or sh-YTHDF2. (H), The stability of Siah2 mRNA was detected by quantitative RT-PCR in CCA cells transfected with sh-NC or sh-YTHDF2 upon $10 \mathrm{mg} / \mathrm{mL}$ actinomycin D treatment. (I),RNA immunoprecipitation assay detected the interaction between YTHDF2 and RNA in CCA cells. RNA enrichment was measured by quantitative RT-PCR. RIP with non-specific IgG was set as control. Western blot of YTHDF2 showed equal amount of input YTHDF2 protein in two groups. (J), Bioinformatics predicted that Siah2 may produce m6A methylation at multiple sites. (K), Immunoblotting of YTHDF2 with cell lysate(Ly.),full-length biotinylated-Siah2(\#1),the Siah2 CDS region with or without m6A motif mutation(\#2,\#3), the Siah2 $3^{\prime}-$ UTR region with or without m6A motif mutation(\#4,\#5), and beads only(NC)in CCA cells Mean \pm SEM of three independent experiments. ${ }^{*} P<0.05$. 
We further tested whether inhibition of SIAH2 affected PD-L1 protein stability by blocking de novo protein synthesis with $\mathrm{CHX}$. As seen in Figure 3C, depletion of Siah2 increased the protein stability of PD-L1 in CCA cells. In contrast, increased Siah2 decreased PDL1 protein level and PDL1 protein stability, whereas mRNA level was not altered in the Siah2 increased group (Figures 3D-F). Moreover, the proteasome inhibitor MG132 rescued the effect of Siah2 on PD-L1, suggesting that Siah2 promotes proteasomal degradation of PD-L1 (Figure 3G). These results strongly suggest that Siah2 promotes PD-L1 proteasomal degradation.

\section{Siah2 Physically Interacts With PD-L1 and Augments the K63-Linked Ubiquitination of PD-L1 in CCA}

We tested whether Siah2 may interact with PD-L1 in CCA. As shown in Figures 4A, B, co-immunoprecipitation assays evidenced that endogenous and exogenous Siah2 specifically interact with PDL1. Thus increased Siah2 in CCA cells enhanced the ubiquitination of PD-L1 in CCA (Figure 4C). We also observed that Siah2 promoted the K63-linked ubiquitination of PD-L1 but not K48linked ubiquitination (Figure 4D). These data suggest that Siah2 physically interacts with PD-L1 and increases the K63-linked ubiquitination of PD-L1 in CCA.

\section{In Vitro Siah2 Enhanced Antitumor T-Cell Immunity}

PD-L1 on tumor cells binds to PD-1 on activated T cells, leading to exhaustion and apoptosis of $\mathrm{T}$ cells, which has been identified as a key process in tumor cell-mediated immune escape (24). To test whether Siah2 may regulate antitumor T-cell immunity via regulating PD-L1, we performed in vitro T-cell-mediated killing assays in a co-culture, in which activated HPBMC were cultured in the presence of human CCA cell lines. HPBMC were activated and expanded with anti-CD3/CD28 antibodies and IL-2, before co-culturing with tumor cells. Lymphocytes used in the study consisted of $60 \% \mathrm{CD}^{+} \mathrm{T}$ cells (Figure $\mathbf{5 A}$ ). CCA cells with LVSiah2 were more susceptible to T-cell killing (Figure 5B). The $\mathrm{CD}^{+}$cell proportion of HPBMC decreased upon co-culturing with the sh-Siah2 cells group, compared with the sh-NC cells group, and increased upon co-culturing with the LV-Siah2 cell group, compared with the LV-NC cells group (Figures 5C, D). Furthermore, the number of apoptotic tumor cells increased in LV-Siah2 cells following HPBMC co-culturing (Figure 5E) Moreover, the mRNA levels of PRF1 (perforin 1), GZMB (granzyme B), GNLY (granulysin), and IFN- $\gamma$ in PBMCs decreased following co-culturing with LV-Siah2 cells, and increased upon co-culturing with shSiah2 cells (Figures 5F, G).
A

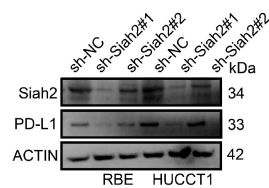

D

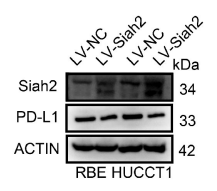

$\mathbf{F}$
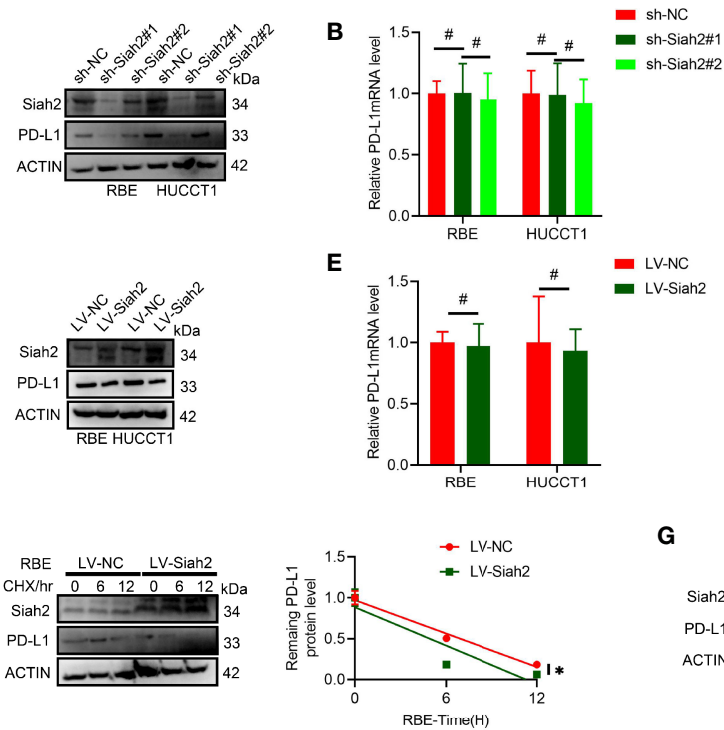

G
C
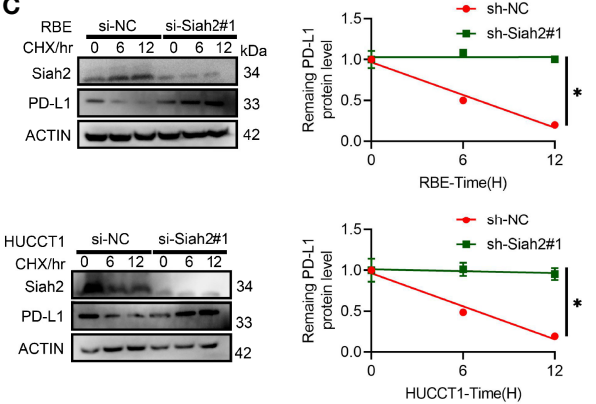
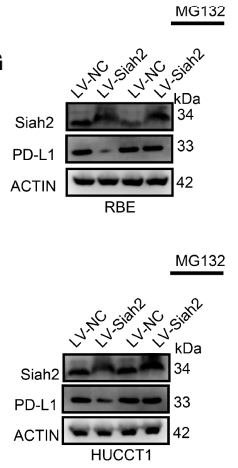

FIGURE 3 Siah2 regulates PD-L1 stability in CCA. (A, B), Depletion of Siah2 increases the protein level but not the mRNA level, of PD-L1 in CCA cells. (C), Depletion of Siah2 increases the protein stability of PD-L1 under CHX treatment. (D, E), Overexpression of Siah2 decreases the protein level, but not the mRNA level, of PD-L1 in CCA cells. (F), Overexpression of Siah2 decreases the protein stability of PD-L1 under CHX treatment. (G), The protein level of Siah2 was evaluated by Western blot in CCA cells with LV-NC or LV-MEITL14 upon MG132 treatment. Mean \pm SEM of three independent experiments. ${ }^{*}$ < 0.05 ; ${ }^{*} \mathrm{P}>0.05$. 


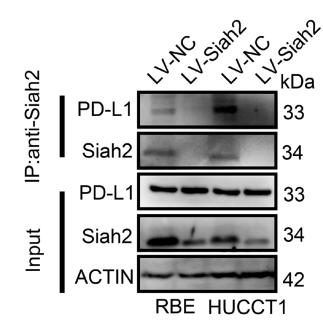

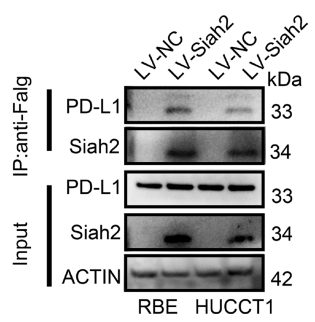

C

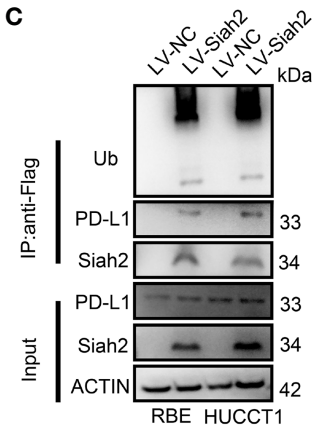

D

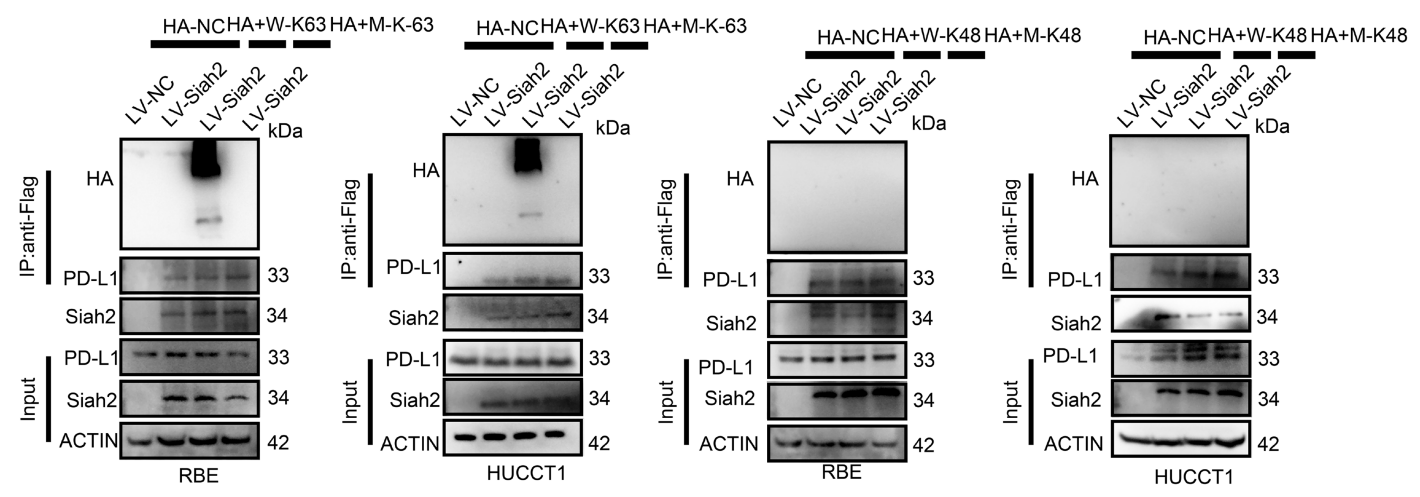

FIGURE 4 | Siah2 physically interacts with PD-L1 and increases the K63-linked ubiquitination of PD-L1 in CCA. (A), CCA cells were transfected with HA-Siah2 and Flag-PD-L1 for $36 \mathrm{~h}$. Immunoprecipitation was performed with anti-Siah2 and analyzed by immunoblotting. (B), CCA cells were transfected with HA-Siah2 and FlagPD-L1 for $36 \mathrm{~h}$. Immunoprecipitation was performed with anti-HA and analyzed by immunoblotting. (C), CCA cells with LV-NC/LV-Siah2 were treated with MG132 for $8 \mathrm{~h}$ before harvesting, after which cells were harvested in lysis buffer. Supernatants were incubated with the appropriate antibodies overnight. Ubiquitin-modified proteins were analyzed with anti-Ub antibodies. (D), CCA cells with LV-NC/LV-Siah2 were treated with MG132 for $8 \mathrm{~h}$ before harvesting, after which cells were harvested in lysis buffer. Supernatants were incubated with the appropriate antibodies overnight. Ubiquitin-modified proteins were analyzed with anti-lysine 48(K48)linked polyubiquitination or K63-linked polyubiquitination.

Taken together, these data suggested that Siah2 deficiency in tumor cells enhanced T-cell-mediated antitumor activity and vice versa.

\section{In Vivo Siah2 Enhanced Antitumor T-Cell Activity}

To verify the potential in vivo effect of Siah2 on CCA progression, we subcutaneously transplanted a xenograft in immunodeficient NCG mice in LV-NC/LV-Siah2 group and did not find a significant difference (Figures 6A-C), indicating that Siah2 exerted a marginal in vivo effect on CCA cell growth. Moreover, the PD-L1 protein level was downregulated by LVSiah2 in xenografts (Figure 6D). Since we lack of a murine CCA cell line, we further transplanted a subcutaneous xenograft of LV-NC/LV-Siah2 CCA cells in HPBMC-transferred immunodeficient NCG mice. HPBMC were pre-activated and expanded with anit-CD3/CD28 antibodies and IL-2 for $12 \mathrm{~d}$ before transferring, and the percentage of $\mathrm{CD}^{+} \mathrm{T}$ cells reached $80 \%$. The tumor size in the LV-Siah2 group was significantly smaller than that of the LV-NC group in HPBMC-transferred NCG mice (Figures 6E-G). As expected, the percentage of human $\mathrm{CD}^{+}$cell in HPBMCs was higher in the LV-Siah2 group than that in the LV-NC group (Figure 6H).

\section{Siah2 Enhanced Antitumor T-Cell Activity in a PD-L1-Dependent Manner}

Flow cytometry detected the membranous distribution of PD-L1 on the primary CCA\#1 and CCA\#2 cells, indicating a high membranous distribution of PD-L1 on PCCA\#2 and absence of PD-L1 membranous distribution on PCCA\#1(Figure 7A). We did not observe a significant change of tumor cell viability between PCCA\#1-shSiah2 and PCCA\#1-shCtrl cells in coculturing with HPBMC (Figure 7B). The difference of cell susceptibility to T-cell killing between PCCA\#2-LV-NC and PCCA\#2-LV-Siah2 was reversed by blocking PD-L1 with pembrolizumab (Figures $\mathbf{7 C}, \mathbf{D}$ ), indicating that $\mathrm{PD}-\mathrm{L} 1$ is required for Siah2-mediated tumor resistance to $\mathrm{T}$-cell antitumor activity.

\section{The Correlation Between METTL14, Siah2, and PD-L1 in Clinical CCA Specimens}

We investigated the clinical correlation between METL14, Siah2, and PD-L1 protein expression. We confirmed a negative correlation between Siah2 and PD-L1 protein level, a negative correlation between METL14 and Siah2, and a positive correlation between METL14 and PD-L1 in 30 human CCA tumor tissues by 


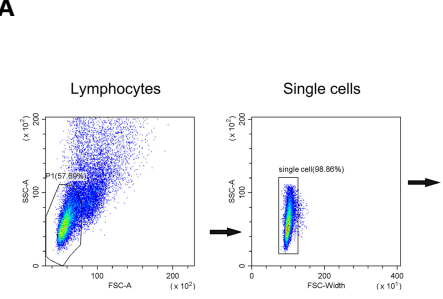

C
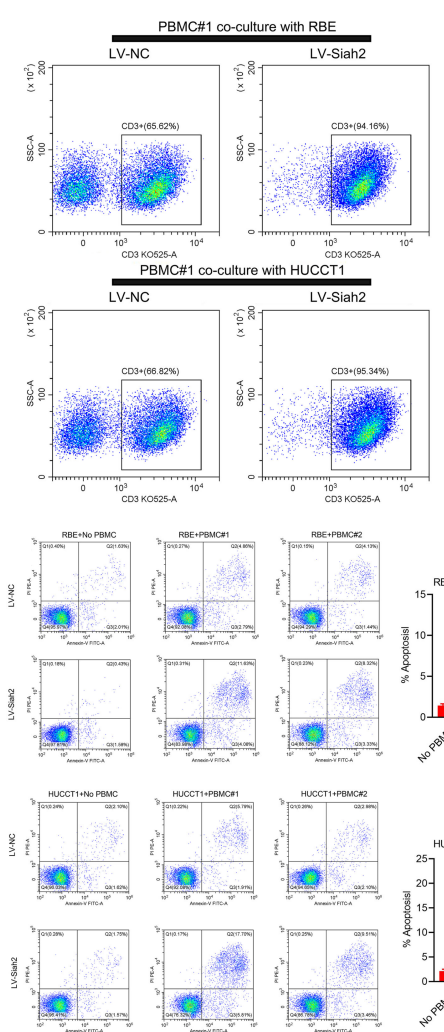

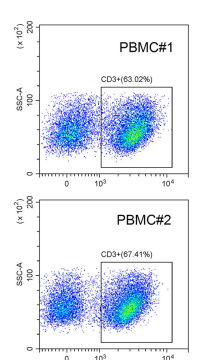

B

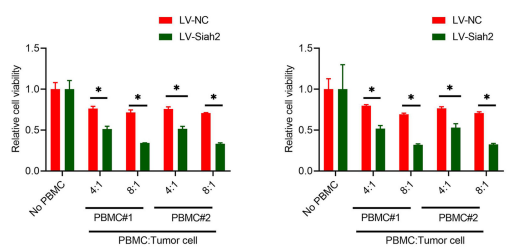

D
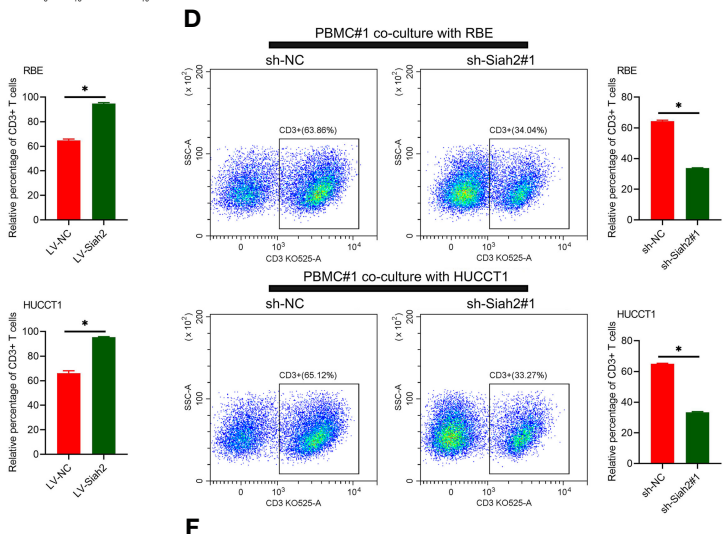

$\mathbf{F}$

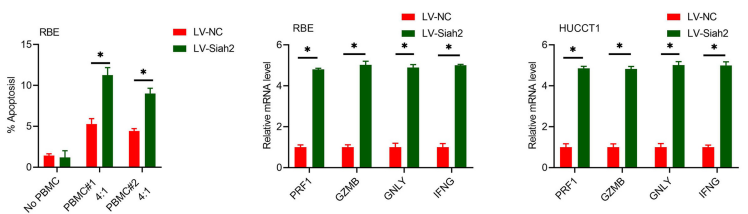

G
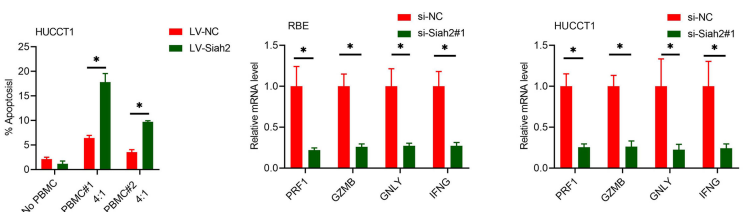

FIGURE 5 | Siah2 enhanced in vitro antitumor T-cell activity. (A), Gating strategy and T cell percentage of activated PBMC. (B), CCK8 assay detected the killing of tumor cells by activated HPBMC. CCA cells were co-cultured in the presence or absence of HPBMC for $24 \mathrm{~h}$. Data were normalized to their respective no HPBMC controls. (C, D), Activated HPBMC (\#1, \#2) were co-cultured with LV-NC/LV-Siah2 (C) or sh-NC/sh-Siah2 (D) for three days at the ratio of HPBMC to tumor cell number of 4 to 1 . The percentage of CD3 in HPBMC was determined by FCM. Representative plots of $\mathrm{CD}^{+} \mathrm{T}$ cells are shown. Data were normalized to the control group. (E), Activated HPBMC (\#1, \#2) were co-cultured with CCA with LV-NC/LV-Siah2 cells for $24 \mathrm{~h}$ at the ratio of HPBMC to tumor cell number of 4 to 1 . HPBMC were collected and stained with PE-Annexin $V$ and subjected to FCM analysis. The percentage of apoptotic cells was analyzed. (F, G), Quantitative RT-PCR was performed to detect PRF1 (perforin-1), GZMB (granzyme), GNLY (granulysin), and IFNG (IFN- $\gamma$ ) in activated HPBMC co-cultured with CCA cells with LV-NC/LV-Siah2 (G) for the indicated time or with si-NC/si-Siah2 cells for $48 \mathrm{~h}$. The ratio of HPBMC to tumor cell number was 4 to 1 . Mean \pm SEM of three independent experiments. ${ }^{*} \mathrm{P}<0.05$

Western blot (Figure 8A). Next, we investigated whether Siah2 expression was associated with anti-PD-L1/PD1 immunotherapy response. We collected six specimens from patients before receiving anti-PD1 immunotherapy, and evaluated the therapeutic responses three months later [2/6 Stable Disease (SD), 4/6 Progressive Disease (PD); Figure 8B]. IHC detection of Siah2 and PD-L1 indicated that SD specimens displayed low Siah2 expression and PD-L1-positive expression on CCA tumor cells, and PD specimens displayed high Siah2 expression and PD-
L1-negative expression (Figure 8C). These data showed that high expression of Siah2 may be an indicator for anti-PD-L1/PD1 immunotherapy response.

\section{DISCUSSION}

The low responsiveness of anti-PD-1/PD-L1 immunotherapy highlights the requirement of complete understanding of the 

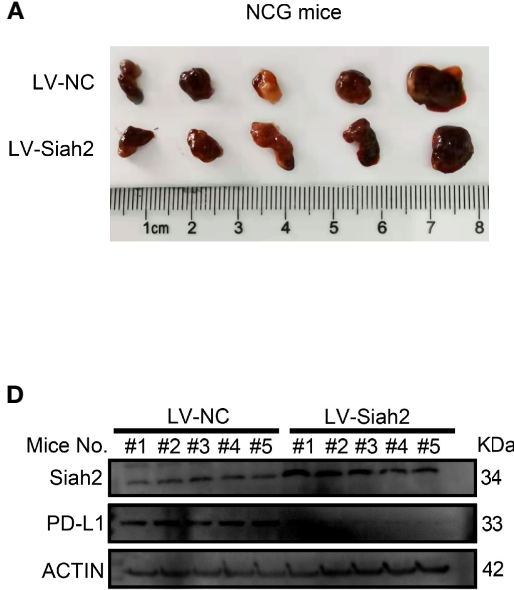

B

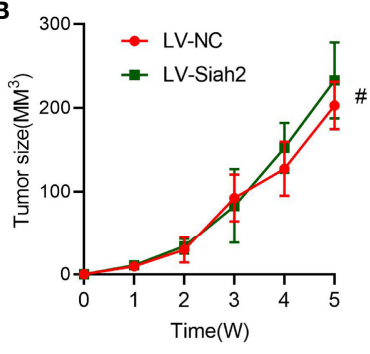

C

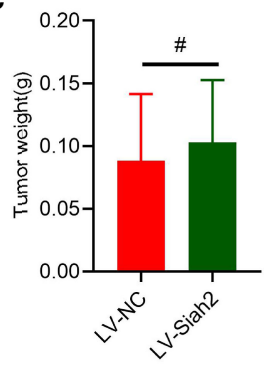

E
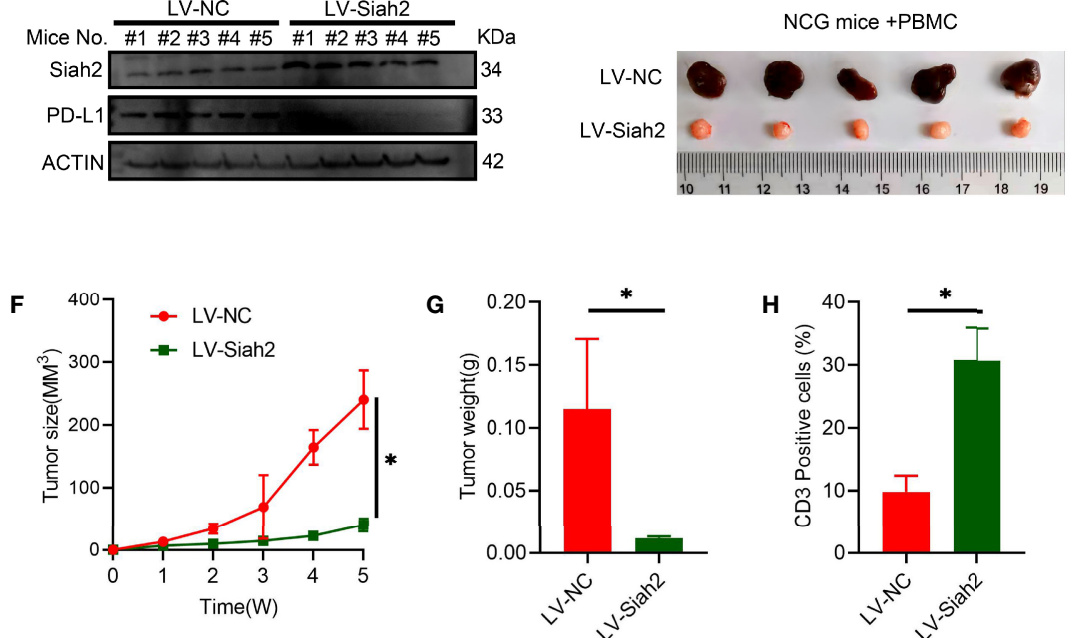

FIGURE 6 | Siah2 enhanced in vivo antitumor T-cell activity. (A-C), CCA cells with LV-NC/LV-Siah2 were injected subcutaneously into the right flank of NCG mice to obtain tumor xenografts. Tumor volume (B) and tumor weight (C) were then measured. $\mathrm{n}=5$. ns, non-significant. Unpaired $t$ test. (D), Western blot analysis detected PD-L1 level in the subcutaneous xenografts described in (A-C). (E-H), CCA cells with LV-NC/LV-Siah2 were injected subcutaneously into the right flank of NCG mice, which were adoptively transferred with activated HPBMC. (E-G), Images of subcutaneous xenografts $(\mathbf{E})$, tumor volume (F), and tumor weight (G) are shown. $N=5$. (H), FCM detected the percentage of human $C D 3^{+}$T cells in HPBMC of NCG mice. $n=5$. Mean \pm SEM of three independent experiments. ${ }^{*} P<0.05$; ${ }^{\#} \mathrm{P}>0.05$.

$\mathrm{PD}-\mathrm{L} 1$ regulation mechanism $(27,28)$. In this study, we reported for the first time a novel m6A modification of MEETL14-Siah2PD-L1 axis in CCA. We found that Siah2 was regulated by an m6A modification in the mRNA, and the main m6A enzyme controlling Siah2 mRNA levels was METTL14. The significant correlation between METTL14 and Siah2 was confirmed in clinical CCA specimens by Western blotting and IHC. We also demonstrated the increased m6A modification on the 3'UTR region of the METTL4-induced PD-L1 mRNA in CCA cells. It has been documented that m6A modification affects mRNA stability, splicing, nuclear export, and/or translation of target mRNAs, depending on the distinct proteins that recognize them [e.g., YTHDF1, YTHDF2, YTHDF3 (6)]. We have shown that decreasing Siah2 mRNA levels by METTL14 were mainly due to the accelerated degradation of Siah2 mRNA, depending on the m6A reader protein YTHDF2, thereby decreasing Siah2 protein level. However, we did not evaluate whether m6A modifications affect the translation efficiency of Siah2, and worth further discussion. To the best of our knowledge, this is the first study to report the involvement of m6A modification in Siah2 dysregulation in cancer.
The activity of PD-1/PD-L1 is complex, since it is modulated by multiple processes, including gene transcription, posttranscriptional modifications, posttranslational modifications (PTMs), and exosome transport (29). PTMs (e.g., glycosylation, phosphorylation, ubiquitination, palmitoylation, SUMOylation, and acetylation) have been demonstrated to play an essential role in the modulation of protein stabilization and protein-protein interactions of the PD-1/PDL1 axis (30-32). Siah2 is known to play an important role in tumorigenesis and cancer progression (18). Numerous studies have determined that Siah2 promotes tumorigenesis in a variety of human malignancies (18). However, several studies revealed that Siah2 exhibited tumor suppressor function by promoting the proteasomemediated degradation of several oncoproteins, suggesting that Siah2 could exert its biological function depending on different stages of tumor development (18). Moreover, Siah2 is subject to complex regulation, especially the phosphorylation of Siah2 by a variety of protein kinases to regulate its stability and activity (18). However, the role of Siah2 in regulating tumor immunoenvironment in CCA, and the underlying molecular mechanism of this potential Siah2-regulated PD-L1 protein level has not been yet elucidated. We showed that Siah2 physically interacts with PD-L1 and increases K63-linked 

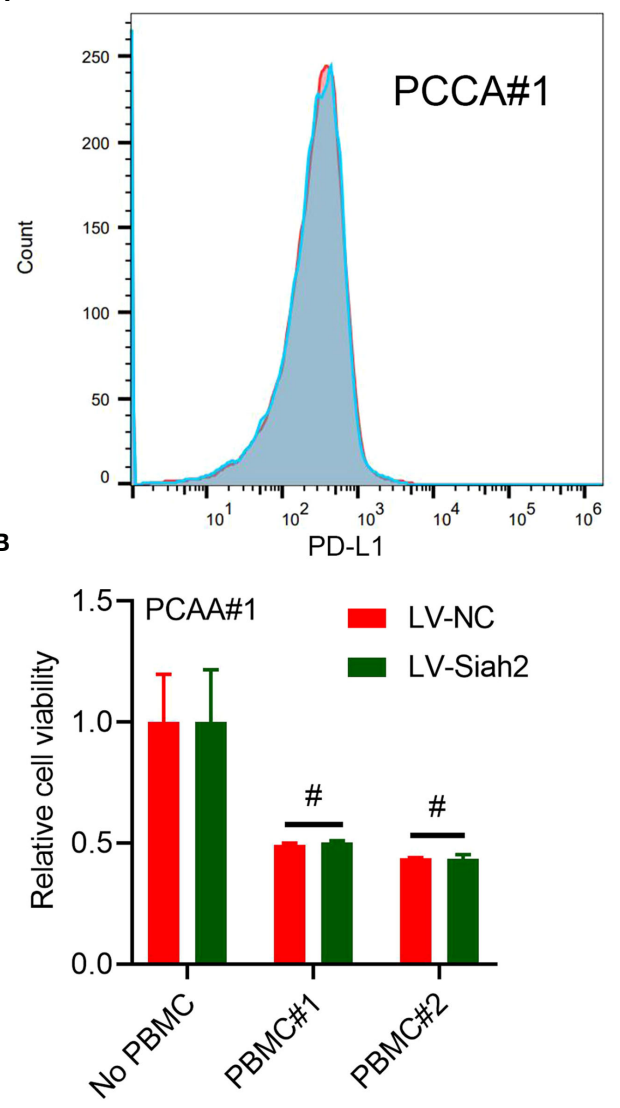

D

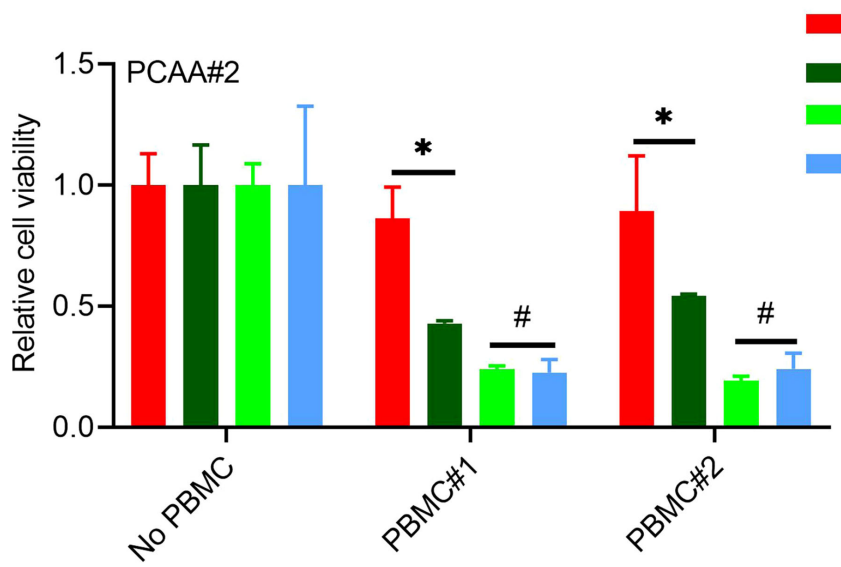

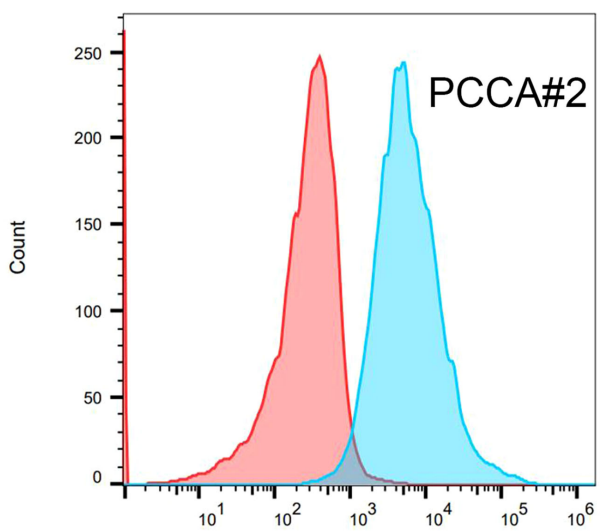

C

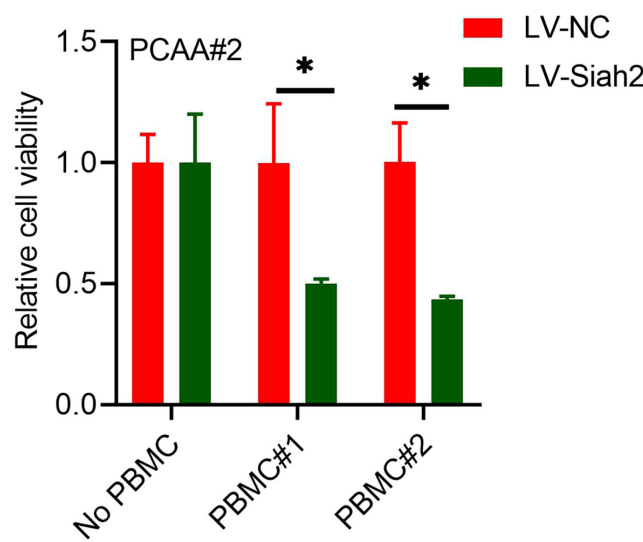

FIGURE 7 | Siah2 enhanced antitumor T-cell immunity in a PD-L1-dependent manner. (A), FCM detected the cell membranous PD-L1 level of PCCA\#1 and \#2. (B, C), CCK8 assay detects the killing of PCAA\#1 LV-NC/LV-Siah2 by activated HPBMC in PCCA\#1 and PCCA\#2 cells. The ratio of PBMC to tumor cell number was 4 to $1, n=3$. (D), CCK8 assay detected the killing of tumor cells by activated HPBMC after Pembrolizumab or 10 Mg/mL IgG treatment. PCAA\#2-LV-NC/LVMEITL14 were co-cultured in the presence or absence of HPBMC for $24 \mathrm{~h}$ at the ratio of HPBMC to tumor cell number of 4 to $1, \mathrm{n}=3$. Mean \pm SEM of three independent experiments. ${ }^{*} \mathrm{P}<0.05$; ${ }^{\#} \mathrm{P}>0.05$.

ubiquitination of PD-L1 in CCA. This finding expands the cognitive scope of the regulation mechanism of PD-L1 protein levels. To our knowledge, this is the first report concerning the role of Siah2 regulating PD-L1 protein level by K63-linked ubiquitination in
CCA, and our results suggest the clinical significance of Siah2-PDL1 axis in CCA Immunotherapy.

In the present study, we demonstrated that tumor-intrinsic Siah2-PD-L1 axis inhibited T cell-mediated cytotoxicity. The 


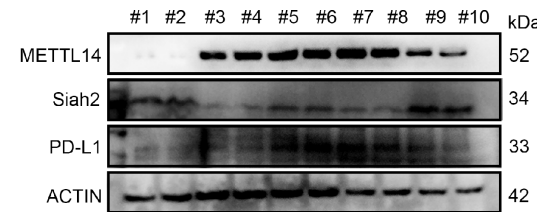

SD-Patient\#1

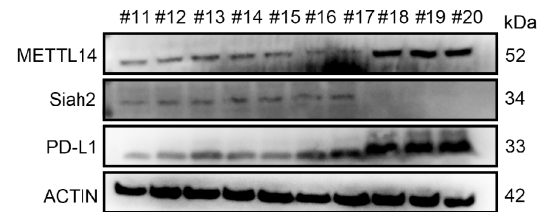

SD-Patient\#3

B
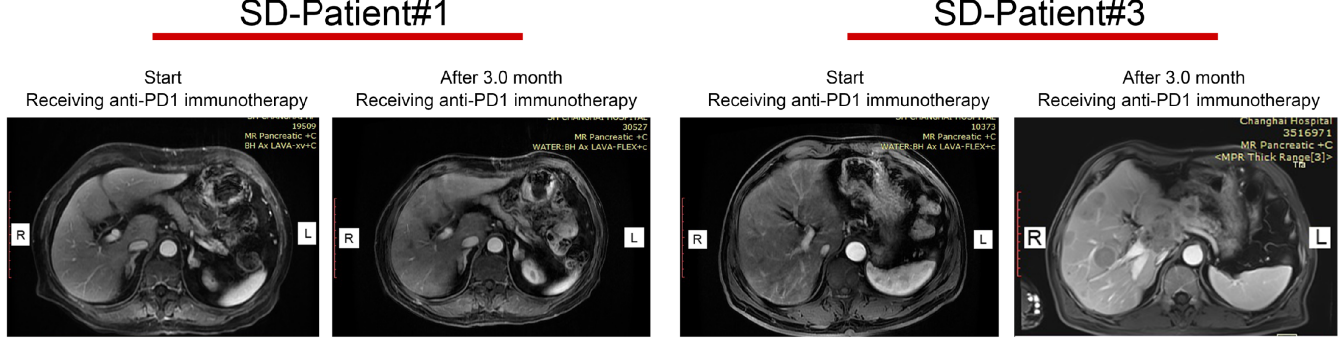

C

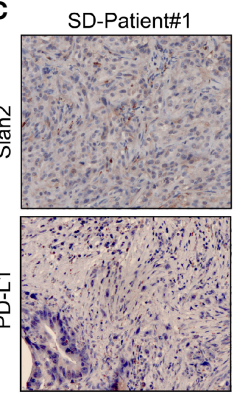

PD-Patient\#1

PD-Patient\#2
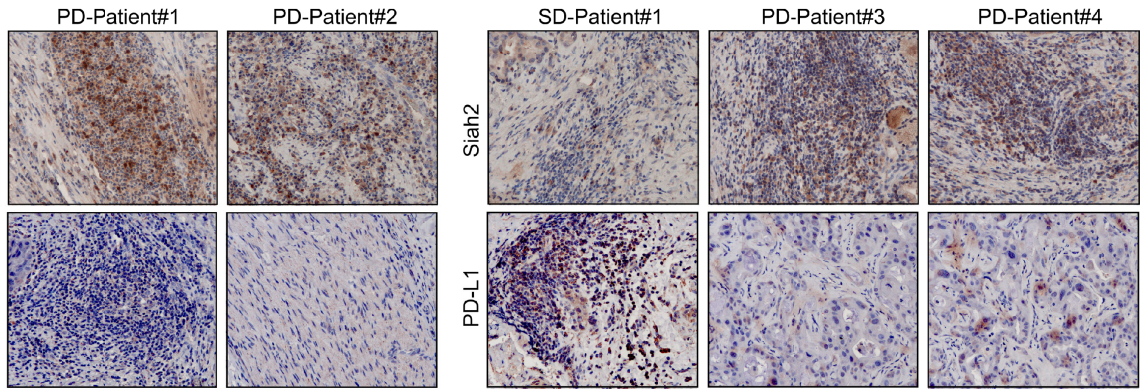

FIGURE 8 | Correlation between MEITL14, Siah2, and PD-L1 in clinical CCA specimens. (A, B), Western blot analysis detected METTL14, Siah2, and PD-L1 protein levels in clinical CCA tumor samples. (B), CT imaging of SD\#1-CCA Patient and PD\#3-CCA Patient. (C), Siah2 and PD-L1 expression in the tumor area of CCA tissues detected by $I H C$. Magnification X 200. PD, progressive disease; SD, stable disease.

lack of a murine CCA cell line restricted our study on tumorimmune microenvironment to optimize immunotherapy in CCA. Therefore, we co-cultured activated HPBMC with human ICC cells in vitro and in vivo to investigate if the inhibitory effect of Siah2 on non-specific T-cell immunity was PD-L1-dependent. For deeper understanding of Siah2 role in ICC tumor immune microenvironment, we used CD $34^{+}$ humanized NCG mice models, which have the same composition of immune cell subsets, especially regarding myeloid cells, which are considered to be a better preclinical model $(33,34)$. By applying CD34+ humanized NCG mice model and PCCA cell model analysis, we further found that Siah2 enhanced in vivo antitumor T-cell immunity in a PD-L1dependent manner, unveiling a complex role of Siah2 in the tumor-immune microenvironment.

In addition, although the use of PD-L1 IHC assays for predicting anti-PD-1/PD-L1 immunotherapy response was approved in some cancers [e.g., pembrolizumab in non-small cell lung cancer (35)], most cancers showed inconsistencies between PD-L1 immunohistochemistry readout and response (36). PD-L1 predictive role is confounded by technical issues and by discrepancies in scoring systems $(36,37)$. N-linked glycosylation of PD-L1 blocks its recognition by IHC but Lee et al. improved the PD-L1 detection method by removing glycosylation (38). Combination of PD-L1 detection with other biomarkers (e.g., tumor mutational burden, tumor-infiltrating lymphocytes, and cytotoxic gene signatures) is also promising (39). We suggest that the addition of Siah2 might have addictive predictive value than PD-L1 IHC alone.

In conclusion, our study demonstrated that Siah2 is a direct target of METTL14 and m6A modification. Mechanically, METTL14 enhances the m6A modification on 3'UTR region of Siah2 mRNA and mainly accelerates the degradation of Siah2 mRNA in an YTHDF2-dependent manner, thereby reducing Siah2 protein level. Siah2 physically interacts with PD-L1 and increases the K63-linked ubiquitination of PD-L1. Siah2 may enhance antitumor T-cell immunity through the Siah2-PD-L1 axis in CCA. Studies on specimens from patients before receiving anti-PD1 immunotherapy suggested that tumors with low Siah2 expression pattern might be more sensitive to anti-PD1 immunotherapy (Figure 9). Taken together, our study unveils the molecular mechanism of M6A-Siah2-PD-L1 axis in CAA, and extends the understanding of Siah2 in tumor-immune microenvironment and immunotherapy. However, this study still has some drawbacks and there is also knowledge worthy of further study, such as we lack a large-scale clinical cohort to verify the expression of mettl14, siah2, and PD-L1 and their correlation with clinical indicators and prognosis. 


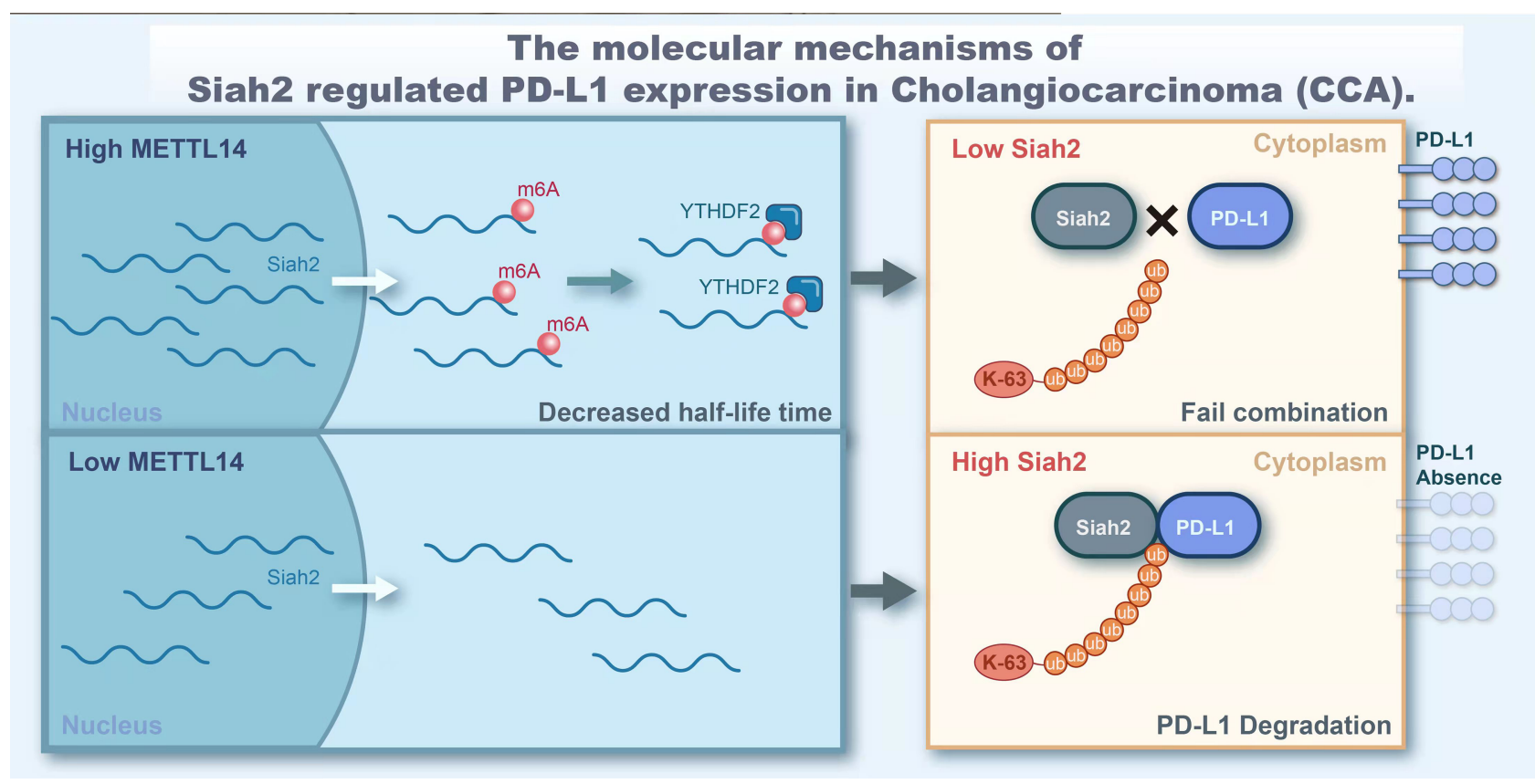

FIGURE 9 | The molecular of Siah2 regulated PD-L1 expression in Cholangiocarcinoma (CCA).

\section{DATA AVAILABILITY STATEMENT}

The raw data supporting the conclusions of this article will be made available by the authors, without undue reservation.

\section{ETHICS STATEMENT}

Tissue specimens were obtained from the Eastern Hepatobiliary Surgery Hospital (Shanghai, China) and the Changhai Hospital (Shanghai, China). The patients/participants provided their written informed consent to participate in this study. All animal experiments were conducted in conformity with conformity with $\mathrm{NIH}$ and the Second Affiliated Hospital of Nanchang University, Servicebio Animal Welfare guidelines and approved by Wuhan Servicebio Technology Co., Ltd., China. For experiments using human samples, written informed consent was obtained from patients. Written informed consent was obtained from the individual(s) for the publication of any potentially identifiable images or data included in this article.

\section{AUTHOR CONTRIBUTIONS}

$\mathrm{HZ}, \mathrm{Z}-\mathrm{gW}$, and Y-pT performed data analysis and interpretation of results. All authors participated in planning and performing the experiments. LF, FZ, and $\mathrm{BL}$ conceived and planned the experiments. All authors contributed to the article and approved the submitted version.

\section{FUNDING}

This work was supported by National Natural Science Foundation of China (No.82160578, No.81760438).

\section{ACKNOWLEDGMENTS}

We extend our gratitude to Edit Springs (https://www. editsprings.com/) for the expert linguistic services provided.

\section{SUPPLEMENTARY MATERIAL}

The Supplementary Material for this article can be found online at: https://www.frontiersin.org/articles/10.3389/fimmu.2022.845193/ full\#supplementary-material 


\section{REFERENCES}

1. Banales JM, Cardinale V, Carpino G, Marzioni M, Andersen JB, Invernizzi P, et al. Expert Consensus Document: Cholangiocarcinoma: Current Knowledge and Future Perspectives Consensus Statement From the European Network for the Study of Cholangiocarcinoma (ENS-CCA). Nat Rev Gastroenterol Hepatol (2016) 13(5):261-80. doi: 10.1038/nrgastro. 2016.51

2. Rizvi S, Gores GJ. Pathogenesis, Diagnosis, and Management of Cholangiocarcinoma. Gastroenterology (2013) 145(6):1215-29. doi: 10.1053/j.gastro.2013.10.013

3. Razumilava N, Gores GJ. Cholangiocarcinoma. Lancet (2014) 383 (9935):2168-79. doi: 10.1016/S0140-6736(13)61903-0

4. Sirica AE, Gores GJ, Groopman JD, Selaru FM, Strazzabosco M, Wei Wang X, et al. Intrahepatic Cholangiocarcinoma: Continuing Challenges and Translational Advances. Hepatology (2019) 69(4):1803-15. doi: 10.1002/hep.30289

5. Qu N, Bo X, Li B, Ma L, Wang F, Zheng Q, et al. Role of N6-Methyladenosine (M(6)A) Methylation Regulators in Hepatocellular Carcinoma. Front Oncol (2021) 11:755206:755206. doi: 10.3389/fonc.2021.755206

6. Zhou Y, Yang J, Tian Z, Zeng J, Shen W. Research Progress Concerning M(6)A Methylation and Cancer. Oncol Lett (2021) 22(5):775. doi: 10.3892/ol.2021.13036

7. Zhang Z, Zhang C, Luo Y, Zhang G, Wu P, Sun N, et al. RNA N(6) -Methyladenosine Modification in the Lethal Teamwork of Cancer Stem Cells and the Tumor Immune Microenvironment: Current Landscape and Therapeutic Potential. Clin Transl Med (2021) 11(9):e525. doi: 10.1002/ctm2.525

8. Gu C, Shi X, Dai C, Shen F, Rocco G, Chen J, et al. RNA M(6)A Modification in Cancers: Molecular Mechanisms and Potential Clinical Applications. Innovation (N Y) (2020) 1(3):100066. doi: 10.1016/j.xinn.2020.100066

9. Dong L, Cao Y, Hou Y, Liu G. N(6) -Methyladenosine RNA Methylation: A Novel Regulator of the Development and Function of Immune Cells. J Cell Physiol (2021) 1-17. doi: 10.1002/jcp.30576

10. Qin S, Mao Y, Wang H, Duan Y, Zhao L. The Interplay Between M6a Modification and Non-Coding RNA in Cancer Stemness Modulation: Mechanisms, Signaling Pathways, and Clinical Implications. Int J Biol Sci (2021) 17(11):2718-36. doi: 10.7150/ijbs.60641

11. Chen L. Co-Inhibitory Molecules of the B7-CD28 Family in the Control of TCell Immunity. Nat Rev Immunol (2004) 4(5):336-47. doi: 10.1038/nri1349

12. Wolchok JD. PD-1 Blockers. Cell (2015) 162(5):937. doi: 10.1016/j.cell.2015.07.045

13. Rizvi S, Khan SA, Hallemeier CL, Kelley RK, Gores GJ. Cholangiocarcinoma Evolving Concepts and Therapeutic Strategies. Nat Rev Clin Oncol (2018) 15 (2):95-111. doi: 10.1038/nrclinonc.2017.157

14. Ahn S, Lee JC, Shin DW, Kim J, Hwang JH. High PD-L1 Expression Is Associated With Therapeutic Response to Pembrolizumab in Patients With Advanced Biliary Tract Cancer. Sci Rep (2020) 10(1):12348. doi: 10.1038/ s41598-020-69366-4

15. Zou W, Wolchok JD, Chen L. PD-L1 (B7-H1) and PD-1 Pathway Blockade for Cancer Therapy: Mechanisms, Response Biomarkers, and Combinations. Sci Transl Med (2016) 8(328):328rv4. doi: 10.1126/scitranslmed.aad7118

16. Sun C, Mezzadra R, Schumacher TN. Regulation and Function of the PD-L1 Checkpoint. Immunity (2018) 48(3):434-52. doi: 10.1016/j.immuni.2018.03.014

17. Ji L, Jiang B, Jiang X, Charlat O, Chen A, Mickanin C, et al. The SIAH E3 Ubiquitin Ligases Promote Wnt/ $\beta$-Catenin Signaling Through Mediating Wnt-Induced Axin Degradation. Genes Dev (2017) 31(9):904-15. doi: 10.1101/gad.300053.117

18. Li K, Li J, Ye M, Jin X. The Role of Siah2 in Tumorigenesis and Cancer Therapy. Gene (2021) 809:146028. doi: 10.1016/j.gene.2021.146028

19. Xu D, Li C. Regulation of the SIAH2-HIF-1 Axis by Protein Kinases and Its Implication in Cancer Therapy. Front Cell Dev Biol (2021) 9:646687. doi: $10.3389 /$ fcell.2021.646687

20. Knauer SK, Mahendrarajah N, Roos WP, Krämer OH. The Inducible E3 Ubiquitin Ligases SIAH1 and SIAH2 Perform Critical Roles in Breast and Prostate Cancers. Cytokine Growth Factor Rev (2015) 26(4):405-13. doi: 10.1016/j.cytogfr.2015.04.002

21. Krämer OH, Stauber RH, Bug G, Hartkamp J, Knauer SK. SIAH Proteins: Critical Roles in Leukemogenesis. Leukemia (2013) 27(4):792-802. doi: 10.1038/leu.2012.284

22. Qi J, Pellecchia M, Ronai ZA. The Siah2-HIF-FoxA2 Axis in Prostate Cancer New Markers and Therapeutic Opportunities. Oncotarget (2010) 1(5):379-85. doi: 10.18632/oncotarget.171
23. Nakayama K, Qi J, Ronai Z. The Ubiquitin Ligase Siah2 and the Hypoxia Response. Mol Cancer Res (2009) 7(4):443-51. doi: 10.1158/1541-7786.MCR-08-0458

24. Sanmamed MF, Chen L. A Paradigm Shift in Cancer Immunotherapy: From Enhancement to Normalization. Cell (2018) 175(2):313-26. doi: 10.1016/ j.cell.2018.09.035

25. Qiu X, Yang S, Wang S, Wu J, Zheng B, Wang K, et al. M(6)A Demethylase ALKBH5 Regulates PD-L1 Expression and Tumor Immunoenvironment in Intrahepatic Cholangiocarcinoma. Cancer Res (2021) 81(18):4778-93. doi: 10.1158/0008-5472.CAN-21-0468

26. Zheng $\mathrm{H}$, Yang $Y$, Hong YG, Wang MC, Yuan SX, Wang ZG, et al. Tropomodulin 3 Modulates EGFR-PI3K-AKT Signaling to Drive Hepatocellular Carcinoma Metastasis. Mol Carcinog (2019) 58(10):1897907. doi: $10.1002 / \mathrm{mc} .23083$

27. 3Mou L, Tian X, Zhou B, Zhan Y, Chen J, Lu Y, et al. Improving Outcomes of Tyrosine Kinase Inhibitors in Hepatocellular Carcinoma: New Data and Ongoing Trials. Front Oncol (2021) 11:752725. doi: 10.3389/fonc.2021.752725

28. Ringelhan M, Pfister D, Connor T, Pikarsky E, Heikenwalder M. The Immunology of Hepatocellular Carcinoma. Nat Immunol (2018) 19(3):22232. doi: 10.1038/s41590-018-0044-z

29. Hu X, Wang J, Chu M, Liu Y, Wang ZW, Zhu X. Emerging Role of Ubiquitination in the Regulation of PD-1/PD-L1 in Cancer Immunotherapy. Mol Ther (2021) 29(3):908-19. doi: 10.1016/j.ymthe.2020.12.032

30. Hsu JM, Li CW, Lai YJ, Hung MC. Posttranslational Modifications of PD-L1 and Their Applications in Cancer Therapy. Cancer Res (2018) 78(22):634953. doi: 10.1158/0008-5472.CAN-18-1892

31. Meng F, Su Y, Xu B. Rho-Associated Protein Kinase-Dependent Moesin Phosphorylation is Required for PD-L1 Stabilization in Breast Cancer. Mol Oncol (2020) 14(11):2701-12. doi: 10.1002/1878-0261.12804

32. Bailly C, Vergoten G. N-Glycosylation and Ubiquitinylation of PD-L1 do Not Restrict Interaction With BMS-202: A Molecular Modeling Study. Comput Biol Chem (2020) 88:107362. doi: 10.1016/j.compbiolchem.2020.107362

33. Hegde PS, Chen DS. Top 10 Challenges in Cancer Immunotherapy. Immunity (2020) 52(1):17-35. doi: 10.1016/j.immuni.2019.12.011

34. Zhao Y, Shuen T, Toh TB, Chan XY, Liu M, Tan SY, et al. Development of a New Patient-Derived Xenograft Humanised Mouse Model to Study HumanSpecific Tumour Microenvironment and Immunotherapy. Gut (2018) 67 (10):1845-54. doi: 10.1136/gutjnl-2017-315201

35. Cottrell TR, Taube JM. PD-L1 and Emerging Biomarkers in Immune Checkpoint Blockade Therapy. Cancer J (2018) 24(1):41-6. doi: 10.1097/ PPO.0000000000000301

36. Diggs LP, Hsueh EC. Utility of PD-L1 Immunohistochemistry Assays for Predicting PD-1/PD-L1 Inhibitor Response. Biomark Res (2017) 5:12. doi: 10.1186/s40364-017-0093-8

37. Hirsch FR, McElhinny A, Stanforth D, Ranger-Moore J, Jansson M, Kulangara K, et al. PD-L1 Immunohistochemistry Assays for Lung Cancer: Results From Phase 1 of the Blueprint PD-L1 IHC Assay Comparison Project. J Thorac Oncol (2017) 12(2):208-22. doi: 10.1016/j.jtho.2016.11.2228

38. Lee HH, Wang YN, Xia W, Chen CH, Rau KM, Ye L, et al. Removal of NLinked Glycosylation Enhances PD-L1 Detection and Predicts Anti-PD-1/ PD-L1 Therapeutic Efficacy. Cancer Cell (2019) 36(2):168-78.e4. doi: 10.1016/ j.ccell.2019.06.008

39. Tseng YH, Ho HL, Lai CR, Luo YH, Tseng YC, Whang-Peng J, et al. PD-L1 Expression of Tumor Cells, Macrophages, and Immune Cells in Non-Small Cell Lung Cancer Patients With Malignant Pleural Effusion. J Thorac Oncol (2018) 13(3):447-53. doi: 10.1016/j.jtho.2017.10.034

Conflict of Interest: The authors declare that the research was conducted in the absence of any commercial or financial relationships that could be construed as a potential conflict of interest.

The editor $\mathrm{CH}$ declared a shared parent affiliation with the authors HZ, LO, GJ at the time of the review.

Publisher's Note: All claims expressed in this article are solely those of the authors and do not necessarily represent those of their affiliated organizations, or those of the publisher, the editors and the reviewers. Any product that may be evaluated in 
this article, or claim that may be made by its manufacturer, is not guaranteed or endorsed by the publisher.

Copyright (๑ 2022 Zheng, Zheng, Wang, Tao, Huang, Yang, Ouyang, Duan, Zhang, Chen, Xiang, Jin, Fang, Zhou and Liang. This is an open-access article distributed under the terms of the Creative Commons Attribution License (CC BY). The use, distribution or reproduction in other forums is permitted, provided the original author(s) and the copyright owner(s) are credited and that the original publication in this journal is cited, in accordance with accepted academic practice. No use, distribution or reproduction is permitted which does not comply with these terms. 